\title{
DUPLICIDAD PIELOURETERAL Y RIÑÓN INTRATORÁCICO
}

\author{
J.R. BELTRÁN ARMADA, A. SERRANO DURBÁ, B. CORONEL SÁNCHEZ, \\ C. DOMÍNGUEZ HINAREJOS, F. ESTORNELL MORAGUES, F. GARCÍA IBARRA
}

Servicio de Urología Infantil. Hospital Infantil La Fe. Valencia.

Actas Urol Esp. 28 (3): 249-251, 2004

\section{RESUMEN}

\section{DUPLICIDAD PIELOURETERAL Y RIÑÓN INTRATORÁCICO}

La ectopia renal intratorácica es un tipo de ectopia muy rara, representando menos del $5 \%$ de las ectopias renales. La asociación de duplicidad pieloureteral y ectopia intratorácica es infrecuente.

Presentamos el caso de una niña afecta de esta asociación de anomalías, tratándose del primer caso descrito en la literatura revisada.

Se comentan los estudios diagnósticos realizados, así como el tratamiento y evolución de la paciente.

PALABRAS CLAVE: Riñón intratorácico. Diagnóstico. Tratamiento.

\section{ABSTRACT \\ PYELOURETERAL DUPLICATION AND INTRATHORACIC KIDNEY}

Intrathoracic ectopia is an unusual ectopia that represents less than $5 \%$ of renal ectopia. The association of a duplex system and an intrathoracic renal ectopia is very rare.

We report a case of a girl affected from these two anormalyties, being the first case published in the literature reviewed.

Presentation, diagnosis and therapeutic aspects are discussed.

KEY WORDS: Intrathoracic kidney. Diagnosis. Treatment.

$\mathrm{L}^{2}$ a ectopia renal intratorácica, protusión renal parcial o total por encima del diafragma, es un tipo de ectopia renal poco frecuente que representa menos del $5 \%$ de las ectopias renales ${ }^{1}$.

La duplicidad pieloureteral se presenta con una incidencia del $0,8 \%^{1}$.

Presentamos el caso de una niña afecta de esta anomalía. Analizamos la clínica, estudios diagnósticos realizados y el tratamiento.

\section{CASO CLÍNICO}

Niña que nace en la 36 semana de gestación, sin ningún hallazgo patológico en el screening prenatal, con parto vía vaginal y presentación cefálica. En la exploración física tras el nacimiento se aprecia abolición del murmullo vesicular en los 2/3 inferiores del campo pulmonar izquierdo y presencia masa abdominal dependiente de fosa renal izquierda con peloteo posterior.

En radiografía de tórax y gammagrafía pulmonar se objetiva hipoplasia pulmonar izquierda.

Se realiza ecografía abdominal no detectando presencia del riñón izquierdo por lo que se realiza exploración ecográfica torácica, apreciando riñón intratorácico izquierdo con duplicidad pieloureteral. Se observa asimismo atrofia parenquimatosa severa de la cortical del pielón superior y uréter tortuoso y dilatado llegando a vejiga en 
situación normal. El hemirriñón inferior presenta leve ectasia con cortical conservada. Riñón derecho normal.

Se practica urografía intravenosa (Fig. 1), TAC helicoidal (Fig. 2) y RMN (Fig. 3), objetivándose riñón izquierdo en situación intratorácica, probablemente debido a hernia diafragmática, con dilatación muy severa del uréter correspondiente al hemirriñón superior y atrofia del parénquima. Pielón inferior y riñón derecho normales. El riñón izquierdo presenta vascularización anómala, dependiendo la del pielón superior de la arteria pulmonar y la del pielón inferior de la aorta. Vascularización renal derecha normal.

La gammagrafía renal muestra un riñón izquierdo anormalmente alto con buena captación del trazador en su pielón inferior y anulación funcional del superior.

Con el diagnóstico de duplicidad pieloureteral izquierda con riñón intratorácico, anulación del pielón superior y hernia diafragmática se procedió a nefroureterectomía polar superior izquierda

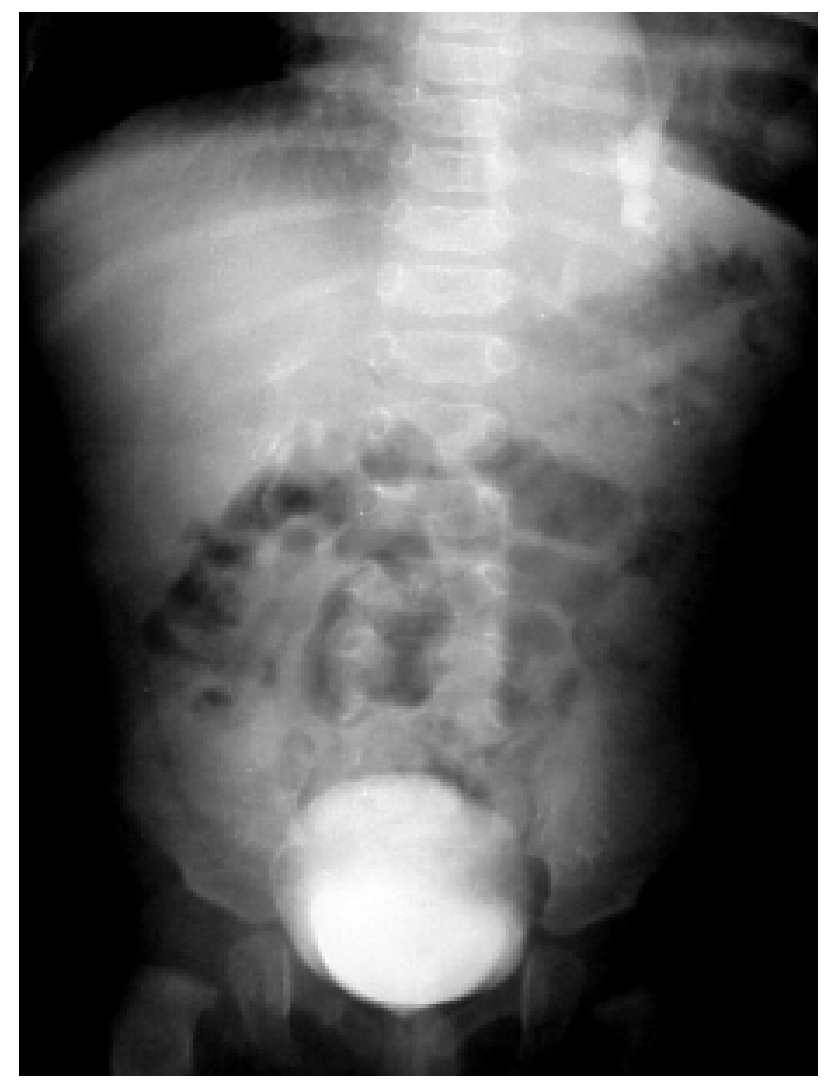

FIGURA 1. UIV: riñón izquierdo de localización intratorácica. por vía toraco-abdominal, movilización renal, pexia en retroperitoneo y plicatura diafragmática.

El post-operatorio se desarrolló sin complicaciones.

Los controles radiológicos a los 6 meses con ecografía, UIV y DMSA, muestran un riñón localizado en fosa renal izquierda con morfología y funcionalidad normal. A los 3 años de seguimiento en controles ecográficos y con DMSA, permanecen estables tanto la morfología como la funcionalidad renal.

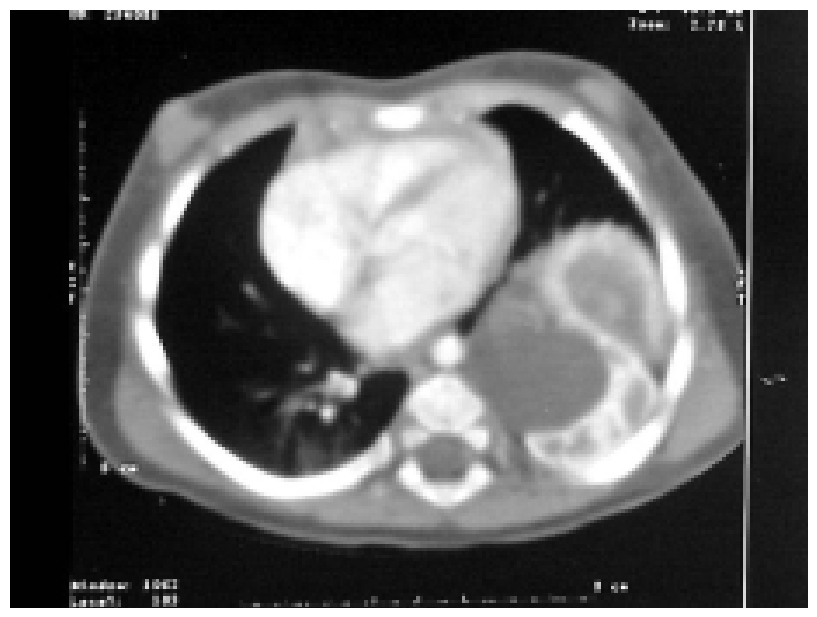

FIGURA 2. TAC: riñón intratorácico e hipoplasia pulmonar.

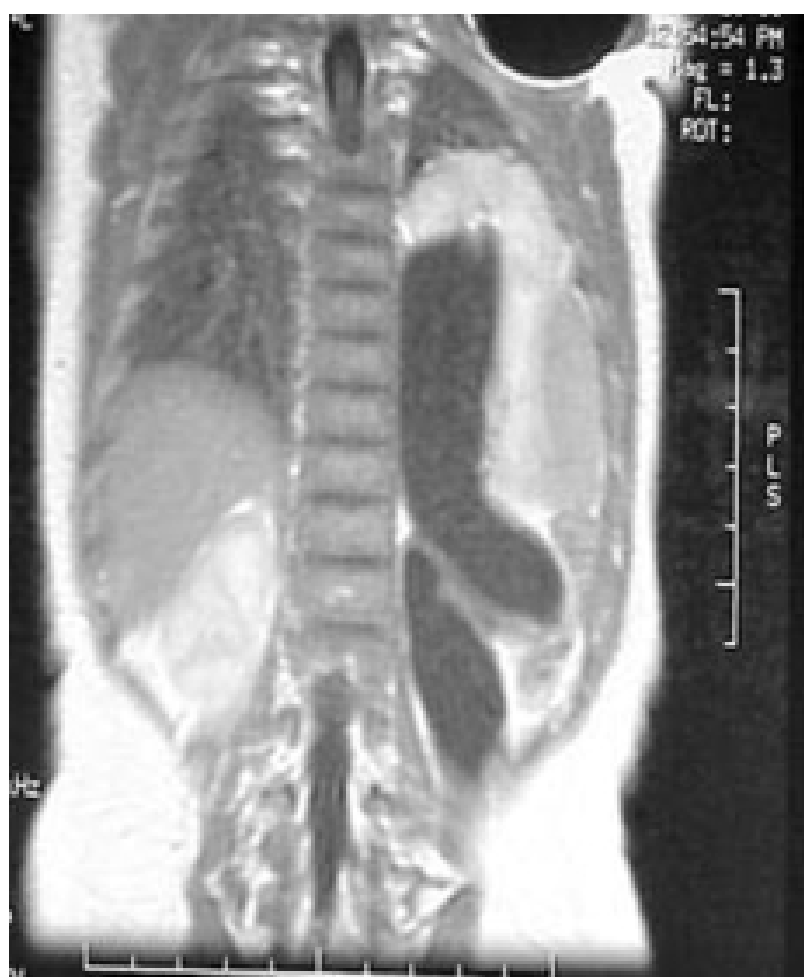

FIGURA 3. RMN: ectasia ureteral del hemirriñón superior. 


\section{DISCUSIÓN}

El riñón intratorácico congénito es una ectopia rara representando apenas el 5\% de los casos. Parece existir un ligero predominio del lado izquierdo en proporción 1,5/1, siendo más frecuente en hombres que en mujeres $(3 / 1)^{2}$. No se conoce con certeza la etiología de este tipo de ectopia, aunque se postula que el retraso en el cierre intrauterino del esbozo diafragmático puede permitir el ascenso renal por encima de su situación anatómica normal ${ }^{1}$. En los estudios angiográficos el origen de la arteria renal es normal en la mayoría de los casos, aunque en algunos, existe irrigación de origen más craneal. El riñón se localiza en la parte posterior del mediastino y normalmente ha completado el proceso de rotación. Se localiza habitualmente en la parte posterolateral del diafragma, en el hiato (orificio de Bochdalek), ya que en esta zona el diafragma presenta menor densidad. El lóbulo inferior del pulmón adyacente puede resultar hipoplásico como consecuencia de la compresión renal ${ }^{4}$, como sucede en nuestro caso.

El uréter es más largo para alcanzar el riñón, aunque su desembocadura en la vejiga es nor$\mathrm{mal}^{5}$. La glándula suprarrenal se ubica en su posición eutópica en la mayoría de los pacientes. No se han descrito anomalías asociadas en otros órganos y la presencia de otras malformaciones de la vía urinaria es poco frecuente. En la literatura consultada no se describe ningún caso de duplicidad completa asociada ${ }^{5}$.

La sintomatología de estos pacientes es escasa. Los síntomas pulmonares son sumamente raros, aunque ante la sospecha de una hipoplasia pulmonar está indicado el estudio para descartar esta entidad ${ }^{2,6}$. La mayoría de los casos se descubren de forma incidental al practicar una radiografía de tórax o una ecografía ${ }^{6}$.

El diagnóstico se realiza mediante radiografía de tórax ${ }^{7}$ que muestra elevación del hemidiafragma y masa ocupante de espacio, redondeada, regular, que se extiende hacia el tórax, cerca de la línea media. La ecografía mostrará la ausencia del riñón en la fosa renal. La U.I.V. y la gammagrafía aclararán definitivamente el diagnóstico. La TAC y RMN pueden realizarse si con las anteriores exploraciones no se llega a un diagnóstico de certeza.
En la actualidad la TAC helicoidal con contraste y su reconstrucción tridimensional, así como la RMN pueden hacer innecesaria la angiografía, lo que es de especial importancia en niños ${ }^{3,4}$. Además, la TAC helicoidal evita el uso de anestesia general.

Si no existe sospecha de reflujo vesicoureteral (ectasia de la vía, ITU o duplicidad) no es necesario la práctica de CUMS.

El tratamiento es conservador, salvo en los casos en que se asocian complicaciones urinarias o pulmonares importantes. En estos casos será precisa la intervención quirúrgica con movilización del riñón y cierre de la hernia diafragmática. En nuestro caso, ante la anulación del pielón superior se optó además por nefroureterectomía parcial.

\section{CONCLUSIONES}

La duplicidad pieloureteral y el riñón intratorácico son patologías infrecuentemente asociadas. Para el diagnóstico, la utilización de la TAC helicoidal permite obviar estudios más invasivos y la necesidad de anestesia general en pacientes pediátricos. El tratamiento de esta patología es el mismo que el de ambas entidades por separado.

\section{REFERENCIAS}

1. CAMPBELL MF.: Renal ectopy. J Urol 1930; 24: 187. 2. RAMOS AJ, SLOVIS TL, REED JO.: Intrathoracic kidney. Urology 1979; 13: 14.

3. GRENADIR DS, LARSEN CR.: Computed tomography of intrathoracic kidney. Urology 1983; 22: 85.

4. JEFFERSON KP, PERSAN RA.: Thoracic kidney: a rare form of renal ectopia. Journal of Urology 2001; 165 (2): 504.

5. HAWWAS N, KOLAWOLE T et al.: Intrathoracic kidneys. Report of 6 cases. Eur Urol 1988; 14: 83.

6. MARCO A, GONZALVEZ J, ANDÚJAR M.: Riñón intratorácico en la infancia: a propósito de un caso. An Esp Pediatr 1993; 38 (5): 455-457.

7. KIRSHENBAUM KS, PURI HC, RAO BR.: Congenital intrathoracic kidney. J Urol 1981; 125 (3): 412-423.

Dr. J.R. Beltrán Armada

Servicio de Urología

Hospital Univ. Dr. Peset

Avda. Gaspar Aguilar, 90

46017 Madrid

(Trabajo recibido el 23 junio de 2003) 